\title{
Erratum to: Incidence and Prevention of Postoperative Venous Thromboembolism: Are They Meaningful Quality Indicators in Japanese Health Care Settings?
}

\author{
Susumu Kunisawa · Hiroshi Ikai • Yuichi Imanaka
}

Published online: 12 October 2011

(C) Société Internationale de Chirurgie 2011

\section{Erratum to: World J Surg}

DOI 10.1007/s00268-011-1229-x

The following are corrections to the original article due to a mistake in identifying VTE prophylaxis:

1. The last sentence in the Results section of the abstract should read:

VTE prophylaxis did not show a relation between DVT and PE despite $82.0 \%$ adherence.

2. In the Conclusions section of the abstract the fourth sentence should read:

However, PE is too rare to be considered a rate-based outcome indicator, and VTE prophylaxis is widely applied to be used as a process indicator.
3. On the fifth page of the article, right column, first paragraph, the second sentence should read:

A high percentage $(82.0 \%, 41,207 / 50,226)$ of the patient underwent VTE prophylaxis. Although nine PE events were observed in the nonprophylaxis group $(9 / 9,019)$, some were observed in the thromboprophylaxis group as well $(105 / 41,207)$.

4. On the sixth page of the article, right column, first paragraph, the first sentence should read:

In contrast, $82.0 \%$ of patients and $88.2 \%$ of patients with DVT and PE in this group underwent prophylactic interventions.

5. Following are revised Figs. 1 and 2 (with the corrected caption):

The online version of the original article can be found under doi: 10.1007/s00268-011-1229-x.

S. Kunisawa $\cdot$ H. Ikai · Y. Imanaka $(\square)$

Department of Healthcare Economics and Quality Management,

Graduate School of Medicine, Kyoto University, Yoshida

Konoe-cho, Sakyou-ku, Kyoto 606-8501, Japan

e-mail: imanaka-y@umin.net 


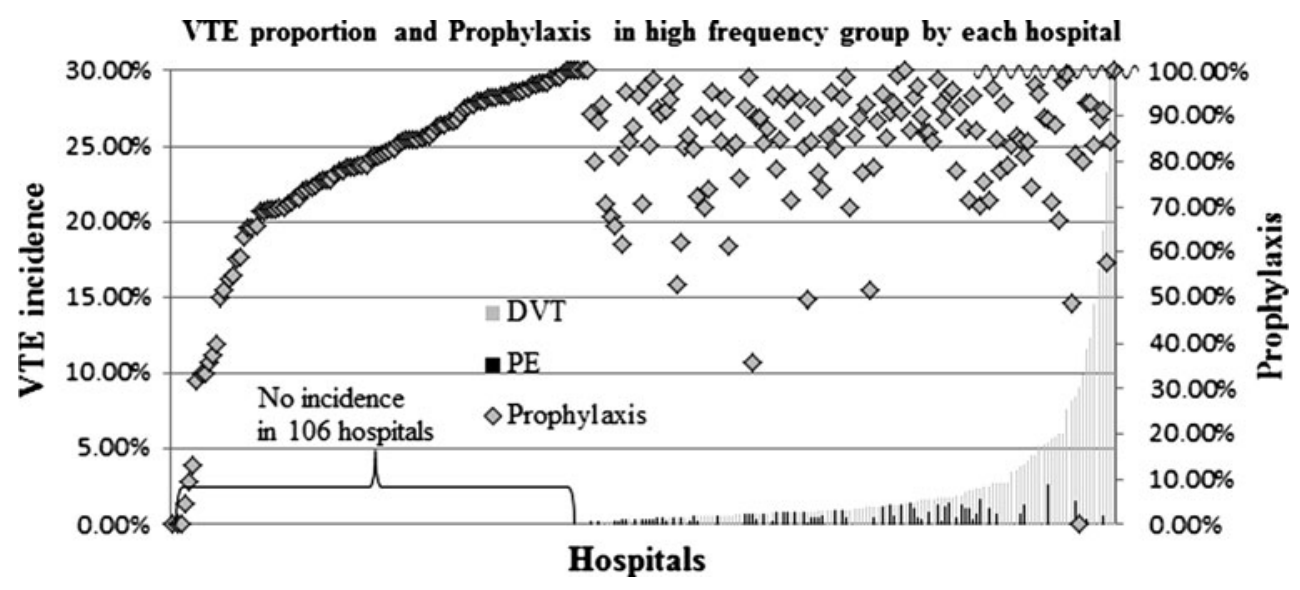

Fig. 1 Venous thromboembolism (VTE) incidence and prophylaxis in high-frequency groups in all of the hospitals. This graph shows the VTE incidence together with deep vein thrombosis (DVT) and pulmonary embolism $(\mathrm{PE})(\mathrm{VTE}=\mathrm{DVT}+\mathrm{PE})$ and VTE prophylaxis. Incidence graph implies a lack of correlation between DVT

Prevention prodedure and

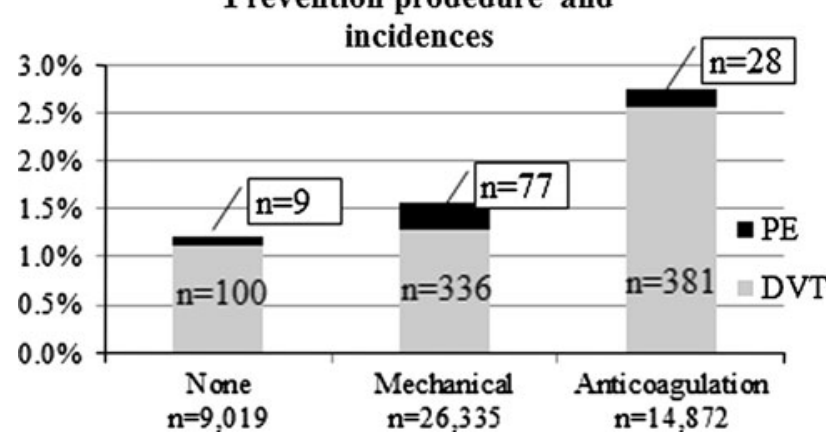

Fig. 2 Incidences of deep vein thrombosis (DVT) and pulmonary embolism (PE) in high-frequency groups in the hospitals. It shows that PE can develop in patients who receive such therapy and those who do not. Although nine PE events were observed in the nonprophylaxis group $(9 / 9,019)$, some were observed in the thromboprophylaxis group as well. incidence and PE incidence. Whereas relatively low-prophylaxis hospitals showed no incidence, those who practiced $100 \%$ prophylaxis did. Each hospital seemed to be highly aware regarding VTE prophylaxis 